\title{
MATHEMATISCHE ZEITSCHRIFT
}

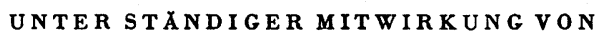 \\ E. K AM KE \\ TUBINGEN \\ R. NEVANLINNA \\ HELSINKI \\ F. K. SCHMIDT \\ HEIDELBERG
}

HERAUSGEGEBEN VON

\section{H. WIELANDT \\ TUUBINGEN}

WISSENSCHA FT LICHER BEIRAT

\begin{abstract}
W. BLA SCHKE L. FEJER A. E. INGHAM H. KNESER
\end{abstract} W. MAGNUS O.PERRON G.PICKERT

70. BAND

BERLIN • GOTTINGEN • HEIDELBERG

S P R I N G E R - V E R L A G 1958/59 


\section{Inhalt des 70. Bandes}

BaChman, G., Geometry in Certain Finite Groups . . . . . . . . . . 466

Barthel, W., Zum Busemannschen und Brunn-Minkowskischen Satz . . . . . . . 407

BEnz, W., $\left(8_{3}, 6_{4}\right)$-Konfigurationen in Laguerre-, Möbius- und weiteren Geometrien 283

Butzer, P. L., Zur Frage der Saturationsklassen singulärer Integraloperatoren . . . 93

Dek Ker, J. C. E., Congruences in Isols with a Finite Modulus . . . . . . . . . . 113

DekKer, J. C. E., The Factorial Function for Isols . . . . . . . . . . . . . . . 250

Dinghas, A., Zur Existenz von Fixpunkten bei Abbildungen vom Abel-Liouvilleschen Typus . . . . . . . . . . . . . . . . . . . . . . . . . . . . 174

FAN, K., On the Equilibrium Value of a System of Convex and Concave Functions 271

Farahat, H. K., and L. Mrrsky, Group Membership in Rings of Various Types . . 231

Fulton, C. M., and D. A. Norton, Non-Existence of Fixed Subspaces under Affine

Transformations . . . . . . . . . . . . . . . . . . . . . . 52

Goes, G., BK-Räume und Matrixformationen für Fourierkoeffizienten . . . . . . 345

GreEn, J. A., On the indecomposable representations of a finite group . . . . . . 430

HоенnкE, H.-J., Über komponierbare Formen und konkordante hyperkomplexc Größen . . . . . . . . . . . . . . . . . . . 1

Hofmans, K. H., Topologische Loops . . . . . . . . . . . . . . . . . . . . 13

Hofmans, K. H., Topologische Loops mit schwachen Assoziativitätsforderungen . . 125

Hofmank, K. H., Topologische Doppelloops . . . . . . . . . . . . . . . . . . 213

$\mathrm{I}_{\mathrm{T} \hat{\mathrm{c}}}, \mathrm{N}$., Normalteiler mehrfach transitiver Permutationsgruppen . . . . . . . . . 165

KA:ScH, F., Uber eine metrische Eigenschaft der S-Zahlen . . . . . . . . . . . . 263

Klein-Barmen, F., Verallgemeinerung des Verbandsbegriffs durch Abschwächung des Axioms der Idempotenz . . . . . . . . . . . . . . . . . . . . . . 83

Klingenberg, W., Eine Kennzeichnung der Riemannschen sowie der Hermiteschen

Mannigfaltigkeiten

KrabBe, G. L., Convolution operators that satisfy the spectral theorem . . . . . . 446

LaUgwirz, D., Eine Bemerkung über konvexe Kurven . . . . . . . . . . . . . 463

MÜller, P. H., Eine neue Methode zur Behandlung nichtlinearer Eigenwertaufgaben 381 
NARAIN, R., A Fourier Kernel . . . . . . . . . . . . . . . . . . . 297

NASToLd, H.-J., Über meromorphe Schnitte komplex-analytischer Vektorraumbündel und Anwendungen auf Riemannsche Klassen. II . . . . . . . . . . . 55

Neumer, W., Kritische Zahlen und bestimmt divergente transfinite Funktionen . . 190

Perron, O., Über zwei ausgeartete Heinesche Reihen und einen Kettenbruch von Ramanujan . . . . . . . . . . . . . . . . . . 245

Rosenberg, A., and D. Zelinsky, Finiteness of the injective hull . . . . . . . 372

Stern, S. K., A Continuous Mapping Defined by a Convex Curve (Addendum). . . 465

Srolt, B., Zur Axiomatik des Brandtschen Gruppoids . . . . . . . . . . . . 156

Thron, W. J., Zwillingskonvergenzgebiete für Kettenbrüche $1+K\left(a_{i l} / 1\right)$, deren eines die Kreisscheibe $\left|a_{2 n-1}\right| \leqq \varrho^{2}$ ist . . . . . . . . . . . . . . . . 310

VOGEL, W. O., Regelflächen in Riemannschen Mannigfaltigkeiten . . . . . . . . . 193

ZARemba, S. K., On Necessary Conditions for the Central Limit Theorem . . . . . 281 


\title{
Über eine metrische Eigenschaft der S-Zahlen
}

\author{
Von \\ FRIEDRICH KASCH
}

\section{Fragestellung, Voraussetzungen}

Eine $Z$ ahl $\xi$ heißt bei der Mahlerschen Klasseneinteilung der transzendenten $Z_{\text {Zahlen }}{ }^{1}$ ) eine $S$-Zahl, wenn es positive Zahlen $\varrho, c_{1}, c_{2}, c_{3}, \ldots$ so gibt, daß für jedes ganzzahlige Polynom $F(x)=\sum_{i} a_{\imath} x^{i}$ vom Grad $n \geqq 1$ und der Höhe $h=\operatorname{Max}_{i}\left(\left|a_{i}\right|\right)$ die folgende Ungleichung gilt:

$$
|F(\xi)| \geqq c_{n} h^{-\varrho n} \text {. }
$$

Auf Grund des Schubfachschlusses ergibt sich bekanntlich, daß bei einer reellen bzw. komplexen $S-Z a h l \xi$ notwendig $\varrho \geqq 1$ bzw. $\varrho \geqq \frac{1}{2}$ sein muB.

In mehreren Arbeiten ist die Frage nach dem Maß der Menge der S-Zahlen in Abhängigkeit von $\varrho$ untersucht worden. Hierzu besagt zunächst ein Ergebnis von A. J. Chintschin [1], daß das Maß der Menge der reellen S-Zahlen mit $\varrho=1$ gleich Null ist. Andererseits zeigte K. MAHLER [6], daß fast alle reellen oder komplexen Zahlen S-Zahlen sind, und zwar bewies er dies für die reellen Zahlen mit $\varrho=4$ und für die komplexen Zahlen mit $\varrho=\frac{7}{2}$. Richtungweisend für die weitere Entwicklung dieser Frage war die Vermutung von K. MAHLER $[6]$, daB fast alle reellen Zahlen $S$-Zahlen mit $\varrho=1+\varepsilon$ und fast alle komplexen Zahlen $S$-Zahlen mit $\varrho=\frac{1}{2}+\varepsilon$ zu beliebig vorgegebenem $\varepsilon>0$ seien.

Das Resultat von K. Mahler wurde zunächst von J. F. Koksma [3] verbessert, der zeigte, daß man die Werte $\varrho=4$ bzw. $\varrho=\frac{7}{2}$ durch 3 bzw. $\frac{5}{2}$ ersetzen kann. Schließlich konnte W. J. LeveQue [5] beweisen, daß es auch für die Werte 2 bzw. $\frac{3}{2}$ gültig ist ${ }^{2}$ ).

Wir bezeichnen mit $\mathfrak{M}_{r}(\varrho)$ die Menge der reellen $S$-Zahlen zu vorgegebenem $\varrho$ in (1) und mit $\mathfrak{M}_{k}(\varrho)$ die entsprechende Menge der komplexen $S$ Zahlen. Sei ferner $\mathfrak{M}_{r}(\varrho, n)$ die Menge der reellen Zahlen $\xi$, die für feste Werte $n$ und $\varrho$ [und für geeignetes $c_{n}=c_{n}(\xi)$ ] der Ungleichung (1) genügen, und sei $\mathfrak{M}_{k}(\varrho, n)$ die entsprechende Menge komplexer Lösungen von (1). Dann gilt offenbar

$$
\mathfrak{M}_{r}(\varrho)=\bigcap_{n=1}^{\infty} \mathfrak{M}_{r}(\varrho, n), \quad \mathfrak{M}_{k}(\varrho)=\bigcap_{n=1}^{\infty} \mathfrak{M}_{k}(\varrho, n)
$$

1) Siehe dazu z. B. [8], Kap. III.

2) Ein zweiter Beweis für dieses Resultat findet sich in [8], Kap. III. 
Sei $\mu(\mathfrak{M})$ das $M a ß$ von $\mathfrak{M}$, dann ist aus maßtheoretischen Gründer $\mu\left(\mathfrak{M}_{r}(\varrho)\right)=1$ äquivalent mit $\mu\left(\mathfrak{M}\left(\varrho_{r}, n\right)\right)=1$ für $n=1,2,3, \ldots$. Die Mahler sche Vermutung besagt dann

$$
\mu\left(\mathfrak{M}_{r}(1+\varepsilon, n)\right)=1
$$

bzw.

$$
\mu\left(\mathfrak{M}_{k}\left(\frac{1}{2}+\varepsilon, n\right)\right)=1
$$

für jedes $\varepsilon>0$ und $n=1,2,3, \ldots$.

Bei dieser Formulierung erhebt sich sofort die Frage, ob man (2) und (3 wenigstens für spezielle Werte von $n$ beweisen könne. Für $n=1$ ist dit Gültigkeit von (2) wohlbekannt und für (3) läßt sie sich sofort verifizieren sogar für den Wert 0 an Stelle $\frac{1}{2}+\varepsilon$. Von J. Kubilyus [4] wurdt (2) auch für $n=2$ bewiesen. Er benutzte dabei wesentlich einen Hilfssat: von J. M. VinogRadov über trigonometrische Summen, also ein verhältnis. mäßig tiefliegendes Hilfsmittel. TH. SCHNEIDER schreibt in seinem Bucl „Einführung in die transzendenten Zahlen “ ${ }^{3}$ ) über das Resultat von J. KUBILYUS „Einen Anfang zum Beweis dieser (Mahlerschen) Vermutung hat viel. leicht J. KubiLyus gemacht ...".

Es dürfte daher von Interesse sein, für das Resultat von J. KubiLyus einen ganz einfachen Beweis zu besitzen. Ein solcher Beweis wird im folgender. mitgeteilt. Wesentlich scheint mir dabei zu sein, daß sich dieser Beweis auch auf den komplexen Fall ausdehnen läßt*). Er liefert dort das Resultat: $\mu\left(\mathfrak{M}_{\dot{k}}\left(\frac{1}{4}+\varepsilon, 2\right)\right)=1$ für jedes $\varepsilon>0$, also ein besseres Ergebnis, als es die Mahlersche Vermutung für beliebiges $n$ besagt.

\section{Hilfssätze}

Hilfssatz 1. Sei $F(x)=a_{0}+a_{1} x+a_{2} x^{2}$ ein Polynom mit ganzrationalen Koeffizienten und verschiedenen Nullstellen $\alpha_{1}, \alpha_{2}$. Mit $D=D(F)$ werde die Diskriminante von $F(x)$ bezeichnet. Dann gilt für jede komplexe Zahl $\xi$ :

$$
\operatorname{Min}\left(\left|\xi-\alpha_{1}\right|,\left|\xi-\alpha_{2}\right|\right) \leq \frac{2}{\sqrt{|D(F)|}}|F(\xi)| .
$$

Beweis. Sei etwa $\operatorname{Min}\left(\left|\xi-\alpha_{1}\right|,\left|\xi-\alpha_{2}\right|\right)=\left|\xi-\alpha_{1}\right|$, dann gilt

$$
\sqrt{|D(F)|}=\left|a_{2}\left(\alpha_{1}-\alpha_{2}\right)\right| \leq\left|a_{2}\right|\left(\left|\alpha_{1}-\xi\right|+\left|\xi-\alpha_{2}\right|\right) \leq 2\left|a_{2}\left(\xi-\alpha_{2}\right)\right| .
$$

Daraus folgt

$$
\left|\xi-\alpha_{1}\right| \leqq \frac{2}{\sqrt{|D(F)|}}\left|a_{2}\left(\xi-\alpha_{1}\right)\left(\xi-\alpha_{2}\right)\right|=\frac{2}{\sqrt{\mid \overline{D(F) \mid}}}|F(\xi)| .
$$

HilfsSaTZ 2. Die folgende Summation erstrecke sich über alle Polynome $F(x)$ vom Grad 2, der festen Höhe h und mit verschiedenen Nullstellen. Dann gilt mit einer von h unabhängigen Konstanten $\gamma_{0}$ :

$$
\sum \frac{1}{V|D(F)|} \leq \gamma_{0} h
$$

3) [S], S. 83 .

*) Zusalz bei der Korr.: Der Beweis läßt sich auch auf den $p$-adischen Fall übertragen und liefert auch dort das bestmögliche Resultat. 
BEweis. Wegen $|D(F)|=\left|a_{1}^{2}-4 a_{0} a_{2}\right|$ und da mindestens ein Koeffizient gleich $\pm h$ sein muß, gilt

$$
\sum \frac{1}{V|D(F)|} \leqq \underset{\substack{-h \leqq a_{0} \leqq h \\-h \leqq a_{0} \leqq h}}{2} \frac{1}{\left|h^{2}-4 a_{0} a_{2}\right|^{\frac{1}{2}}}+4 \sum_{\substack{-h \leqq a_{1} \leqq h \\-h \leqq a \leqq h}}^{\prime} \frac{1}{\left|a_{1}^{2}-4 h a\right|^{\frac{1}{2}}} ;
$$

die erste Summe besitzt den Faktor 2, da $a_{1}= \pm h$ sein kann und die zweite den Faktor 4 , da $a_{0}= \pm h$ und $a_{2}= \pm h$ sein können. Der Strich am Summenzeichen soll hier wie im folgenden $h^{2}-4 a_{0} a_{2} \neq 0$ bzw. $a_{1}^{2}-4 h a \neq 0$ bedeuten. Betrachten wir jetzt die erste Summe auf der rechten Seite von (5) bei festem $a_{0} \neq 0$. Der Ausdruck $\left|h^{2}-4 a_{0} a_{2}\right|$ durchläuft für $-h \leqq a_{2} \leqq h$ höchstens $2 h+1$ von Null verschiedene Zahlen, von denen je zwei aufeinanderfolgende bis auf höchstens eine Ausnahme alle den Abstand $4\left|a_{0}\right|$ voneinander haben. Die eine Ausnahme kann auftreten, wenn der Ausdruck $h^{2}-4 a_{0} a_{2}$ das Vorzeichen wechselt. Daher gilt

$$
\sum_{-h \leqq a_{2} \leqq h}^{\prime} \frac{1}{\left|h^{2}-4 a_{0} a_{2}\right|^{\frac{1}{2}}} \leqq 2 \sum_{a=0}^{h} \frac{1}{\left(1+4\left|a_{0}\right| a\right)^{\frac{1}{2}}} \leqq 2+\frac{1}{|| a_{0} \mid} \sum_{a=1}^{h} \frac{1}{\sqrt{a}} \leqq 4 \frac{\sqrt{h}}{\sqrt{\left|a_{0}\right|}} .
$$

Damit folgt

$$
\sum_{\substack{h \leqq a_{2} \leqq h \\-h \leqq a_{0} \leqq h}} \frac{1}{\left|h^{2}-4 a_{0} a_{2}\right|^{\frac{1}{2}}} \leqq 4 \sqrt{h} \sum_{\substack{h \leqq a_{0} \leqq h \\ a_{0} \neq 0}} \frac{1}{\sqrt{\left|a_{0}\right|}}+\frac{2 h+1}{h} \leqq 19 h .
$$

Aus (6) entnimmt man ferner für die zweite Summe in (5) bei festem $a_{1}$ :

$$
\sum_{-h \leqq a \leqq h}^{\prime} \frac{1}{\left|a_{1}^{2}-4 h a\right|^{\frac{1}{2}}} \leqq 4
$$

Daher erhält man

$$
\sum_{\substack{-h \leqq a \\-h \leqq h}}^{\prime} \frac{1}{\left|a_{1}^{2}-4 h a\right|^{1}} \leqq 4(2 h+1) \leqq 12 h .
$$

Die Abschätzungen (5), (7) und (8) zusammen liefern die Behauptung (4).

Diese beiden Hilfssätze reichen aus, um das Resultat von J. KubILyus zu beweisen. Die nächsten Hilfssätze werden für den komplexen Fall gebraucht.

Hilfssatz 3. Sei $A(q, m)$ die Anzahl der mod $q$ inkongruenten Lösungen von

$$
x^{2} \equiv m(\bmod q)
$$

und sei $r^{2}$ der größte gemeinsame quadratische Teiler von $q$ und $m$. Dann gilt mit einer von $q$ und $m$ unabhängigen Konstanten $\gamma_{2}$

$$
\left.A(q, m) \leqq \gamma_{2} r q^{\frac{2}{\log \log q} 4}\right)
$$

4) Für $q=1$ ist $q^{\frac{2}{\log \log q}}$ gleich 1 zu setzen; entsprechend im folgenden. 
Beweis. Setzt man $m^{\prime}=\frac{m}{r^{2}}$ und $q^{\prime}=\frac{q}{r^{2}}$, dann erhält man aus jeder Lösung $x$ von (9) eine Lösung $x^{\prime}=\frac{x}{r}$ von

$$
x^{\prime 2} \equiv m^{\prime} \quad\left(\bmod q^{\prime}\right)
$$

Umgekehrt liefert jede Lösung $x^{\prime}$ von (10) eine Lösung $x=r x^{\prime}$ von (9). Da aus $0 \leqq x<q$ ferner $0 \leqq x^{\prime}<{ }_{r}^{q}=r q^{\prime}$ folgt, hat man die Beziehung $A(q, m)=$ $r A\left(q^{\prime}, m^{\prime}\right)$. Ist $q^{\prime}=1$, also $q=r^{2}$, dann gilt $A(q, m)=r$ und die Behauptung ist erfüllt. Im folgenden kann daher $q^{\prime}>1$ vorausgesetzt werden. Ist $q^{\prime}=$ $p_{1}^{e_{1}} \ldots p_{t}^{e_{t}}$ die Primzahlpotenzzerlegung von $q^{\prime}$, dann gilt bekanntlich

$$
A\left(q^{\prime}, m^{\prime}\right)=\prod_{i=1}^{t} A\left(p_{i}^{e_{i}}, m^{\prime}\right) \text {. }
$$

Ist $\left(p_{i}, m^{\prime}\right)=1$, dann hat man im Falle $p_{i} \neq 2: A\left(p_{i}^{e_{i}}, m^{\prime}\right)=2$ und im Falle $p_{i}=2: A\left(p_{i}^{e_{i}}, m^{\prime}\right) \leqq 4$. Sei nun $\left(p_{i}, m^{\prime}\right) \neq 1$, dann muß zunächst $\left(p_{i}^{e_{i}}, m^{\prime}\right)=p_{i}$ gelten, da kein quadratischer Faktor auftreten kann. Ist (10) überhaupt lösbar, dann folgt ferner $e_{i}=1$. Daraus ergibt sich aber $A\left(p_{i}^{e_{i}}, m^{\prime}\right)=A\left(p_{i}, m^{\prime}\right)$ $=1$. Insgesamt folgt $A\left(q^{\prime}, m^{\prime}\right) \leqq 2^{t+1}$, wobei also $t$ die Anzahl der verschiedenen Primteiler von $q^{\prime}$ ist. Sei $d\left(q^{\prime}\right)$ die Anzahl aller positiven Teiler von $q^{\prime}$, dann gilt $2^{t} \leqq d\left(q^{\prime}\right)$. Andererseits besteht die Abschätzung ${ }^{5}$ )

$$
d\left(q^{\prime}\right) \leqq \gamma_{1} q^{\prime} \frac{2}{\log \log q^{\prime}},
$$

mit einer von $q^{\prime}$ unabhängigen Konstanten $\gamma_{1}$. Daher erhält man schließlich

$$
A(q, m)=r A\left(q^{\prime}, m^{\prime}\right) \leqq \gamma_{1} r q^{\frac{2}{\log \log q^{\prime}}} \leqq \gamma_{2} r q^{\frac{2}{\log \log q}},
$$

wobei $\gamma_{2}$ von $q$ und $m$ unabhängig ist $\left.{ }^{6}\right)$.

Hilfssatz $4^{7}$ ). Voraussetzungen wie in Hilfssatz 2. Dann gilt mit won $h$ unabhängigen Konstanten $\gamma_{3}$ und $\tau$ :

$$
\sum \frac{1}{|D(F)|} \leqq \gamma_{3} h^{\frac{\tau}{\log \log h}} .
$$

BEwEIs. Wir haben die Lösungszahl der diophantischen Gleichung

bzw.

$$
h^{2}-4 a_{0} a_{2}=m
$$

$$
\frac{h^{2}-m}{4}=a_{0} a_{2}
$$

5) Siehe z. B. [7], S. 24, Satz 5.2.

6) Die Funktion $x^{\frac{2}{\log \log x}}$ ist für $x \geqq e^{e}$ monoton wachsend. Daher gilt die letzte Ungleichung für $q^{\prime} \geqq e^{e}$. Für die endlich vielen Werte $1<q^{\prime}<e^{e}$ kann die Ungleichung durch Vergrößerung der Konstanten erfüllt werden.

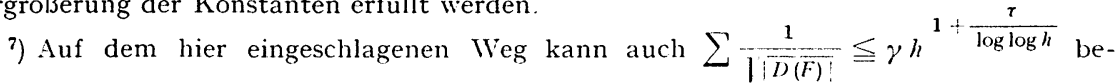
wiesen werden, was an Stelle von Hilfssatz 2 ebenfalls zum Bewcis der endgïltigen Behauptung im reellen Fall ausreicht. 
zu vorgegebenen ganzen Zahlen $h \geqq 1$ und $m$ mit $-3 h^{2} \leqq m \leqq 5 h^{2}$ in ganzen Zahlen $a_{0}, a_{2}$ zu betrachten. Sie ist nach unserer Bezeichnung gleich $4 d\left(\frac{h^{2}-m}{4}\right)$, falls $\frac{h^{2}-m}{4}$ eine von 0 und \pm 1 verschiedene ganze Zahl ist, d.h. falls $m \neq h^{2}$ bzw. $m \neq h^{2} \pm 4$. Setzt man noch $\left|\frac{h^{2}-m}{4}\right|=M$, dann gilt zufolge der schon oben benutzten Abschätzung

Daraus folgt

$$
d\left(\frac{h^{2}-m}{4}\right)=d(M) \leqq \gamma_{1} M^{\frac{2}{\log \log M}} \leqq \gamma_{2} h^{\frac{4}{\log \log \gamma}}
$$

$$
\sum_{\substack{h \leqq a_{0} \leqq h \\-h \leqq a_{2} \leqq h}}^{\prime} \frac{1}{\left|h^{2}-4 a_{0} a_{2}\right|} \leqq 8 \gamma_{2} h^{\frac{4}{\log \log h}} \sum_{\substack{m=1 \\ m \neq h^{2} \\ m \neq h^{2} \pm 4}}^{5 h^{2}} \frac{1}{m}+\frac{6}{h} \leqq \gamma_{4} h^{\frac{5}{\log \log h}}
$$

wobei $\gamma_{4}$ wie auch die noch folgenden Konstanten $\gamma_{5}, \gamma_{6}, \ldots$ von $h$ unabhängig sind.

Sodann haben wir die Lösungszahl der Gleichung

$$
a_{1}^{2}-4 h a=m
$$

unter entsprechenden Voraussetzungen wie oben zu bestimmen. Aus Hilfssatz 3 folgt: Die Anzahl der Lösungen von (13) mit $-h \leqq a_{1} \leqq h$ ist kleiner oder gleich

$$
A(4 h, m) \leqq \gamma_{5} r h^{\frac{2}{\log \log h}} .
$$

Daraus folgt, wenn noch $r=r(m)$ gesetzt wird

$$
\sum_{\substack{-h \leqq a_{1} \leq h \\-h \leqq a \leqq h}}^{\prime} \frac{1}{\left|a_{1}^{2}-4 h a\right|} \leqq 2 \gamma_{5} h^{\frac{2}{\log \log h}} \sum_{m=1}^{5 / 2} \frac{r(m)}{m} .
$$

Es bleibt noch die rechts stehende Summe abzuschätzen. $\mathrm{Zu}$ vorgegebenem $r(m)=k$ kommen darin höchstens die folgenden Glieder vor:

Daraus folgt

$$
\sum_{i=1}^{5 h^{2}} k i \leqq \gamma_{6} \log (h+1) \text {. }
$$

$$
\sum_{m=1}^{5 h^{2}} \underset{m}{r(m)} \leqq d(4 h) \gamma_{6} \log (h+1) \leqq \gamma_{7} h \cdot \stackrel{3}{\log \log h}
$$

und damit erhält man aus (14)

$$
\sum_{\substack{h \leq a_{1} \leqq h \\-h \leqq a \leqq h}}^{\prime} \frac{1}{\left|a_{1}^{2}-4 h a\right|} \leqq \gamma_{8} h^{\frac{5}{\log \log h} .}
$$

Die Abschätzungen (12) und (15) zusammen liefern die Behauptung (11). 


\section{Beweise}

3.1. Wir behandeln zuerst den reellen Fall und zeigen:

$Z u$ beliebig vorgegebenem $\varepsilon>0$ hat die Komplementärmenge von $)_{X_{r}}(1+\varepsilon, 2)$ das Maß Null.

Diese Komplementärmenge ist enthalten in der Menge $\mathfrak{i}$ der reellen Zahlen $\xi$, für die die Ungleichung

$$
|F(\xi)|<h^{-\cdot 2(1+\imath)}
$$

unendlich viele Lösungen in Polynomen $F(x)$ vom Grad 2 hat, und es genügt daher $\mu(\mathfrak{N})=0 \mathrm{zu}$ zeigen. Hat (16) unendlich viele Lösungen, so gibt es auch entweder unendlich viele Lösungen $F(x)$ mit verschiedenen Nullstellen oder unendlich viele Lösungen $F(x)$ mit gleichen Nullstellen. Die Menge der $\xi$, für die (16) unendlich viele Lösungen mit verschiedenen Nullstellen besitzt, nennen wir $\mathfrak{u}$, die Menge der $\xi$, für die (16) unendlich viele Lösungen mit gleichen Nullstellen besitzt, sei $\mathfrak{2}$. Dann gilt $\mathfrak{l}=\mathfrak{l} \cup \mathfrak{2}$.

Behauptung. $\mu(\mathfrak{u})=0$.

Nach der Bezeichnung von Hilfssatz 1 sei Min $\left(\left|\xi-\alpha_{1}\right|,\left|\xi-\alpha_{2}\right|\right)=\left|\xi-\alpha_{1}\right|$, dann folgt aus Hilfssatz 1 und (16):

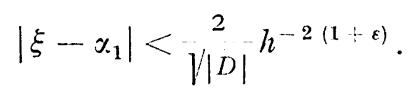

Bezeichnet man zu festem $h$ mit $\mathfrak{u}_{h}$ die Menge der reellen Zahlen $\xi$, zu denen es ein $\alpha_{1} \neq \alpha_{2}$ mit (17) gibt, so gilt wegen Hilfssatz 2

$$
\mu\left(\mathfrak{U}_{h}\right) \leqq 8 h^{-2(1+\varepsilon)} \sum \frac{1}{\| D(F) \mid} \leqq \gamma_{9} h^{-(1+2 \varepsilon)} .
$$

Der Faktor 8 ergibt sich, weil jedes Polynom zwei Nullstellen hat und die Intervallänge aus (17) zweimal genommen werden muß. Für jedes natürliche $r$ gilt offenbar

$$
\| \leqq \bigcup_{h=r}^{\infty} \mathbb{U}_{h} .
$$

Da die Reihe $\sum_{h=1}^{\infty} h^{-(1+2 *)}$ konvergiert, folgt $\mu(1)=0$.

Behauptung. $\mu$ (比) $=0$.

Es mögen jetzt unendlich viele Lösungen $F(x)$ von (16) mit $\alpha_{1}=\alpha_{1.2}$ existieren. Dann kann $F(x)$ in der Form $F(x)=a(b x-c)^{2}$ geschrieben werden und für die Höhen $h$ von $F(x)$ und $h_{1}$ von $b x-c$ gilt $h \geqq h_{1}^{2}$. Daher folgt aus (16)

$$
|b \Sigma-c|<h_{1}^{-2(1: *)} \text {. }
$$

Die Menge der reellen Zahlen $\xi$, die bei festem $h_{1}$ und (sonst) belicbigem $b$ und $c$ diese Gleichung lösen, sei $\aleph_{h_{1}}$. Dann gilt offenbar

$$
\mu\left(-2 h_{1}\right) \leqq 4\left(2 h_{1}+1\right) h_{1}^{-2(1+\varepsilon)} \leqq \gamma_{10} h_{1}^{-(1+2 \varepsilon)} .
$$


liesitzt (16) unendlich viele Lösungen $F(x)=a(b x-c)^{2}$, so besitzt auch (19) unendlich viele Lösungen $b x-c$. Wie im ersten Fall folgt jetzt $\mu(\mathfrak{B})=0$. Damit haben wir $\mu\left(\mathfrak{M}_{r}(1+\varepsilon, 2)\right)=1$ für jedes $\varepsilon>0$ vollständig bewiesen.

3.2. Der Beweis für $\mu\left(\mathfrak{M}_{k}\left(\frac{1}{4}+\varepsilon, 2\right)\right)=1$ für jedes $\varepsilon>0$ wird analog geführt. $\|$ und $\$$ mögen die analoge Bedeutung wie im reellen Fall besitzen. An Stelle von (17) tritt jetzt

$$
\left|\xi-\alpha_{1}\right|<\frac{2}{\sqrt{|D|}} h^{-2\left(\frac{1}{4}+\varepsilon\right)} .
$$

Zufolge von Hilfssatz 4 erhält man daraus

$$
\mu\left(\mathfrak{U}_{h}\right) \leqq 8 \pi h^{-4(1+\varepsilon)} \sum \frac{1}{|D(F)|} \leqq \gamma_{11} h^{-(1+3 \varepsilon)},
$$

wobei jetzt an Stelle der Intervalle bei der Abschätzung von $\mu\left(\mathfrak{l l}_{h}\right)$ in (18) Kreise mit dem Radius $\frac{2}{\sqrt{|D|}} h^{-2(1+\varepsilon)}$ getreten sind. Daraus folgt wieder $\mu(\mathfrak{l l})=0$. Analog zu (19) gilt jetzt

$$
|b \xi-c|<h_{1}^{-2}(1+\varepsilon) .
$$

Diese Ungleichung hat aber bei nichtreellem $\xi=\xi_{1}+i \xi_{2}$ wegen $|b \xi-c| \geqq$ $\left|b \xi_{2}\right| \geqq\left|\xi_{2}\right|$ nur endlich viele Lösungen in linearen Polynomen $b x-c$, woraus sofort $\mu($ Qi $)=0$ folgt.

\section{Schlußbemerkung}

Es soll hier auf zwei Fragen aufmerksam gemacht werden, die sich im Anschluß an unsere Überlegungen stellen. Zunächst wird man fragen, ob sich die hier mitgeteilten Úberlegungen auch auf den Fall $n>2$ ausdehnen lassen. Für Hilfssatz 1 ist das möglich, und man erhält das folgende Resultat, welches eine Verbesserung der entsprechenden Hilfssätze bei I. N. FELDMANN ${ }^{8}$ ) und Th. Schneider ${ }^{9}$ ) darstellt:

Sei $F(x)$ ein ganzzahliges Polynom von einem Grade $n \geqq 2$ ohne mehrfache Nullstellen $\alpha_{1}, \ldots, \alpha_{n}$. Dann gilt für jede Zahl $\xi$

mit

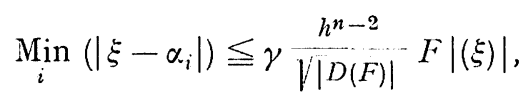

$$
\gamma=(n+1)^{n-2} 2^{\frac{n(3 n-5)}{2}}
$$

Die Möglichkeit, damit zu einer Verbesserung des im allgemeinen Falle bisher besten Resultates von W. J. Leveque zu gelangen, hängt davon ab, dic Summe $\sum_{V \mid \frac{1}{|D(F)|}}$ bzw. $\sum_{|D(F)|}^{1}$ für festen Grad $n$ und feste Höhe $h$ nichttrivial abzuschätzen ${ }^{10}$ ).

Die zweite Frage, die sich hier erhebt, besteht darin, ob ein zu dem anfangs erwähnten Resultat von A. CHrN TSCHIn [1] analoges Resultat auch im komplexen

8) [2], Lemma 5.

9) [8], S. 78 , Hilfssatz 18.

19) Zusalz bei der Korr.: Siehe dazu die demnächst in den Math. Ann. erscheinende Arbeit von B. Volkmann und dem Verf.: Zur Mahlerschen Vermutung über S-Zahlen. 
Falle gilt. In dieser Hinsicht ist

$$
\mu\left(\mathfrak{M}_{k}\left(\frac{n-1}{2 n}, n\right)\right)=0, \quad n=2,3, \ldots
$$

zu vermuten.

\section{Literatur}

[1] Chintschin, A. J.: Zwei Bemerkungen zu einer Arbeit von Herrn Perron. Math. Z. 22, 274-284 (1925). - [2] Feldmann, N. I.: Approximation einiger transzendenter Zahlen I (russ.). Izvestija Akad. Nauk SSSR., Ser. matm. 15, 53-74 (1951). [3] Koksma, J. F.: Uber die Mahlersche Klasseneinteilung der transzendenten Zahlen und die Approximation komplexer Zahlen durch algebraische Zahlen. Monatshefte für Math. und Physik 48, 176-189 (1939). - [4] Kubilyus, J.: Über die Anwendung einer Vinogradowschen Methode auf die Lösung eines Problems aus der metrischen Zahlentheorie (russ.). Doklady Akad. Nauk SSSR. (N. S.) 67, 783-786 (1949). - [5] LeveQue, W. J.: Note on S-Numbers. Proc. Amer. Math. Soc. 4, 189-190 (1953). - [6] MAHLER, K.: Uber das Maß der Menge aller S-Zahlen. Math. Ann. 106, 131-139 (1932). - [7] PrACHAR, K.: Primzahlverteilung. Berlin: Springer 1957. - [8] SChneider, TH.: Einführung in die transzendenten Zahlen. Berlin: Springer 1957.

Heidelberg, Math. Institut der Universität

(Eingegangen am 13. Mai 1958) 